\title{
5G-smart Diabetes: Personalized Diabetes Diagnosis with Human Services Big Data Clouds
}

\section{Narmada}

\begin{abstract}
Ongoing advances in remote systems administration and huge information innovations, for example, $5 G$ systems, medicinal huge information investigation, and the Internet of Things, alongside ongoing improvements in wearable figuring and man-made brainpower, are empowering the advancement and usage of imaginative diabetes checking frameworks and applications. Because of the deep rooted and efficient damage endured by diabetes patients, it is basic to plan powerful strategies for the determination and treatment of diabetes. In light of our far reaching examination, this paper characterizes those strategies into Diabetes 1.0 and Diabetes 2.0, which show insufficiencies as far as systems administration and insight. Consequently, our objective is to structure a manageable, financially savvy, and insightful diabetes finding arrangement with customized treatment. In this paper, we initially propose the 5G-Smart Diabetes framework, which joins the best in class advancements, for example, wearable 2.0, machine learning, and huge information to create complete detecting and investigation for patients experiencing diabetes. At that point we present the information sharing system and customized information examination display for 5G-Smart Diabetes. At last, we construct a 5G-Smart Diabetes testbed that incorporates savvy dress, cell phone, and huge information mists. The trial results demonstrate that our framework can successfully give customized analysis and treatment proposals to patients.
\end{abstract}

Keywords : About four key words or phrases in alphabetical order, separated by commas.

\section{INTRODUCTION}

A wireless sensor network is a network of nodes that togethermeasure the environmental parameters, and establishescommunication between computersand the environment. The nodes of WSN are the sensor nodes and actuator nodes. Along with these nodes, WSN contains gateways and clients. Many sensor nodes deployed arbitrarily near the sensor area and create networks by self-organization. Sensor nodes observe the data to transmit to other nodes by hopping. During transmission, observed data are controlled by many nodes to reach gateway node following the multihop routing, and lastlygo to the management node. Nodes require energy which can supplied by external sources like a battery. These batteries can provideless power. Hence the applications of WSNs are restricteddue to less battery power. Thus, energy becomes a major constraint in WSNs.

Revised Manuscript Received on 14 August, 2019.

* Correspondence Author

V.Narmada, Assistant Professor, Malla Reddy Engineering College for Women (Autonomous), India. narmadavunnam@gmail.com
The network of length varies from several hundred meters to miles is known as access network. This network comprises all the components between the backbone network and the user stations. The backbone network utilizes optical fiberassembly with a largecommunication rate. Hence the access network has become the bottleneck of the complete network. The working of the access network is to coordinate the consumption of channel resources to guarantee the interconnection and transmission of multiple users on the shared channel. Depending on the distance and speed, the present access strategiesaretypified intofour types.

1. wireless local area network (WLAN),

2. wireless metropolitan area network (WMAN),

3. wireless personal area network (WPAN)

4. wireless wide area network (WWAN).

In a WSN,the sensor nodes transmittheir rank to the environmentand gets a rank from other nodes to sense each other.A gateway presents in WSN in order to makea connection to the internet. Then, the sensor nodes form into a linkednetwork based on a particular topology (linear, star, tree, mesh, etc.). To conclude the process, appropriate paths are found on the created network to send the data. The sensor network nodes aretypicallysupplied by batteries, so the broadcast distance of nodes is less. The transmission distance can be around 800 to 1000 meters in the open atmosphere. It will abruptlydrop in a protected indoor atmosphere to some distance. In order to increase the exposure of a network, the network employs multi-hop transmission phase. In this phase the sensor network nodes act as both transmitter and receiver.

The locations of nodes are arbitrary, and they can be relocated, protected and inhibited. The mesh networks havemany gains in flexibility and reliability than other network structures.In smart mesh adhoc networking, the node monitors the adjacent nodes and the signal strength Then it chooses the properadjacentnode for time synchronization and transmits a joining request. Then the adjacent node sends the request to the gateway. The gateway collects the request and allocates network resources for the node. According to the mesh network, the nodes can be set with many transmission tracks to enhance the network reliability. Time synchronized mesh protocol (TSMP) network can provisiode self-organizing network and preserve a network of hundred nodes. 
The concept of the internet of things (IoT) was grown with the development of WSNs. The term internet of things refers to exclusive lyrecognizable objects and their virtual illustrations in an "internet-like" construction. Data brokers contain a thriving business as the assembling of data from several sources and it will bring aboutfresh and mysterious business occasions and latent legal liabilities. TheAdvanced Research Projects Agency Network (ARPANET) presents for so many years, with 200 hosts at universities and research institutes.

Wireless sensor-actuator networks or WSANs are the improved wireless sensor network having powerful and moving actuators. The actuators function with the sensor nodes, and do application-specific actions. To functionrapidly and precisely, an effective and consistent reporting method is critical for the sensors to notify the actuators regarding the atmospheric events. Our transmission algorithm also approves smart priority scheduling that discriminates the event data. Ourmethod is validatedthrough simulations, and the outcomesprove that it attains reliability with less delay.

The locations of nodes are arbitrary, and they can be relocated, protected and inhibited. The mesh networks havemany gains in flexibility and reliability than other network structures.In smart mesh adhoc networking, the node monitors the adjacent nodes and the signal strength. Then it chooses the properadjacentnode for time synchronization and transmits a joining request. Then the adjacent node sends the request to the gateway. The gateway collects the request and allocates network resources for the node. According to the mesh network, the nodes can be set with many transmission tracks to enhance the network reliability. Time synchronized mesh protocol (TSMP) network can provisiode self-organizing network and preserve a network of hundred nodes.

The concept of the internet of things (IoT) was grown with the development of WSNs. The term internet of things refers to exclusivelyrecognizable objects and their virtual illustrations in an "internet-like" construction. Data brokers contain a thriving business as the assembling of data from several sources and it will bring aboutfresh and mysterious business occasions and latent legal liabilities. TheAdvanced Research Projects Agency Network (ARPANET) presents for so many years, with 200 hosts at universities and research institutes.

Wireless sensor-actuator networks or WSANs are the improved wireless sensor network having powerful and moving actuators. The actuators function with the sensor nodes, and do application-specific actions. To functionrapidly and precisely, an effective and consistent reporting method is critical for the sensors to notify the actuators regarding the atmospheric events. Our transmission algorithm also approves smart priority scheduling that discriminates the event data. Ourmethod is validatedthrough simulations, and the outcomesprove that it attains reliability with less delay.

In Wireless Sensor Actuator Networks (WSAN), sensor nodes perform the sensing task and actuator nodes chooseactionsaccording to the sensed event in the field. To guarantee efficient and preciseperformance of WSAN, in this paper, we presented a new Real-Time Coordination and
Routing (RCR) process for WSAN. RCR arranges sensors to create hierarchical clusters and transfersDelay-constrained Energy Aware Routing (DEAR) method. It employs only cluster-heads to direct with sink/actors to prevent the valuable energy resources. The DEAR protocolassimilates the forward tracking and backtracking routing methods to createpaths from source nodes to sink node. In front of the sink in WSAN, it executes the centralized version of DEAR (C-DEAR) to organize with the actors by means of the sink. When there is no sink node, there is a distributed DEAR (D-DEAR) to deliver coordination. Cluster-heads prefer the path among multiple substitute paths to convey the packets to the actuators within the specified delay limitefficiently.

\section{SYSTEM METHODLOGY}

Developing an overlay on a WSAN for data communication prevent data flooding and enhance system scalability, communication speed and energy-efficiency. A well-designed overlay should be energy-efficient in topology maintenance, resilient to node mobility, and enables efficient and reliable routing. A Kautz graph $\mathrm{K}(\mathrm{d}$; k) can help solve the graph connection optimization problem by achieving a tradeoff between the degree and diameter with its minimum degree and relatively shorter diameter. So, Kautz graph is aninexpensive design for WSAN to get the energy-efficiency and real time needs.

For a graph $\mathrm{G}, \mathrm{N}(\mathrm{G})$ and $\mathrm{E}(\mathrm{G})$ denote the number of nodes and edges of the graph, respectively. Graph G's connectivity is the least number of nodes whose elimination results in a disconnected graph.In a Kautz graph $\mathrm{K}(\mathrm{d} ; \mathrm{k})$ with degree d and diameter $\mathrm{k}$, nodes are labeled as (u1...uk). A WSAN overlay involves the consistency between the overlay topology and the under- lying physical topology, which is critical to real-time communication and energy-efficiency.

There are two advantages of the REFER.

REFER is the first technique that incorporates a Kautz graph into the physical structure of a MANET to upholdreliability.

REFER can swiftly and efficiently recognize the differenttracks and their lengths depend on node IDs when routing fails.

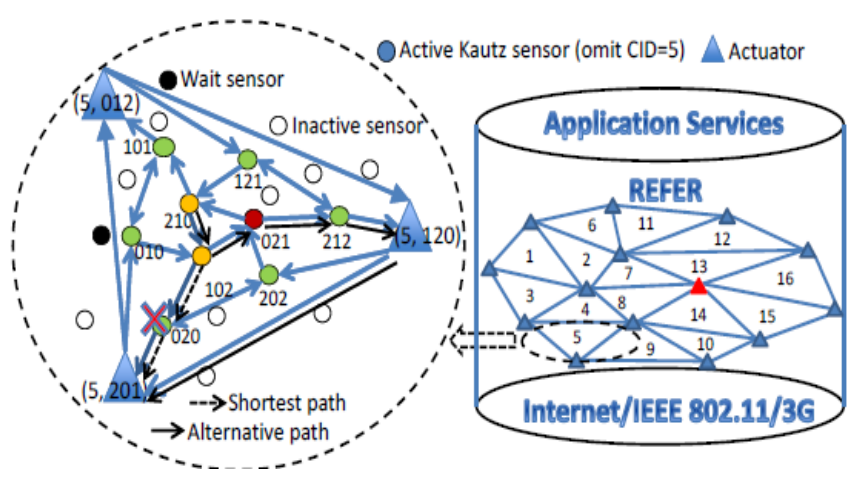

Fig. 2.1: The architecture of the REFER system

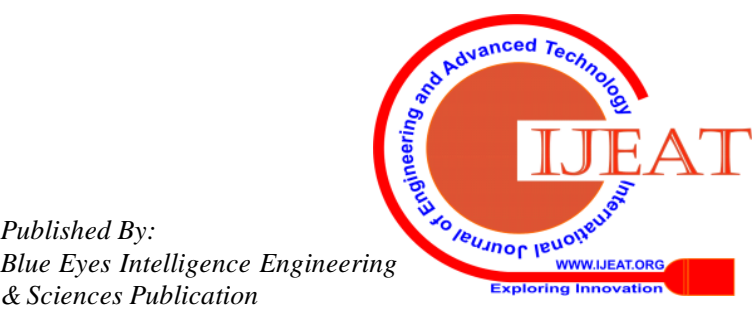


In a sensor network, nodes create a Kautz graph, the upper limit of the coverage area of the sensors is $(2 * r+b) 2=(13 / 4$ r)2. The transmission distance of sensors, $r$ is limited and so the coverage region of the network is restricted. Hence, we require a number of Kautz graph cells with small diameter and degree to cover a large area. Itshows that the density of the sensor nodes in a Kautz cell is large. Hence, a sensor awake/sleep method is required, which can conserve the energy of the sensors and guarantee the connections of Kautz cells.

Actuators and many active sensors in every cell create a Kautz graph and the actuators organize a DHT arrangement.We use resource-rich actuators for the corner vertices of a Kautz graph because they can directly communicate with each other even though they are physically far apart. Although the communication between two nodes in a WSAN is bi-directional, we represent a WSAN as a directed graph $\mathrm{G}(\mathrm{d}, \mathrm{k})$ in order to clearly present the routing. Communication in the other direction can be conducted by simply reversing the direction. The DHT facilitates the information transmission between cells. DHTs are well-known for their scalability and dynamism-resilience.

Each cell contains actuators and sensors. REFER incorporates a Kautz graph into every cell. It is demonstrated that when the diameter $\mathrm{k}$ drops, the number of nodes in Kautz graph $\mathrm{K}(\mathrm{d} ; \mathrm{k})$ reaches the Moore bound. That is, node density in $\mathrm{K}(\mathrm{d} ; \mathrm{k})$ becomes large due to less value of $\mathrm{k}$. Hence, a Kautz graph with small sizeshould be a better option for an overlay to effectively a sensed region. Based on the number of nodes, $\mathrm{n}=(\mathrm{d}+1) \mathrm{dk}-1$ in a WSAN cell and $\mathrm{k}$, the value of $\mathrm{d}$ can be determined. $d$ is the minimum number that produces the value smaller than $n$.

The DHT enables the data communication between cells. DHTs are well-known for their scalability and dynamism-resilience. In addition, without relying on energy-inefficient topological routing or geographic routing, the constructed DHT preserves the physical topology to enable the DHT routing algorithm to communicate data to the target node, over theactuators which are located very close. Thus fast and energy-efficient routing is attained.

An actuator places in variousneighboring cells and has CIDs are not same for different cells. Todecreasethe complexity, we assume that each actuator has the same KID. To ensure the reliability between overlayandphysical arrangement, nodecommunicationisutilized

to decidethenodeIDasthetransmission distance signifies node physical distances. Kautz ID assignment is a task of incorporating a Kautz graph to a cell. It has two stages: actuator ID assignment and sensor ID assignment.

The actuator ID assignment perceives triangles in the neighboring actuators and consecutivelyallots IDs to the actuators. In centralized of assignment, The triangulation algorithm is employed to design actuators to triangles. In the distributed kind, adjacent actuators exchange the information with each other. Initially, one node is chosen as a starting node. The starting node chooses its nextneighbor and constructs an edge to it. To detect the adjacentnode on one side of edge eij connecting nodes, si and sj, these nodes transmit message with each other to find out their shared neighbours. For each shared neighbour skm, the perpendicular bisectors of eikm and ejkm intersect with the perpendicular bisector of eij at a point. Then, the shared neighbour that produces the least distance between such a point and eij is the nearest neighbour (signified by sk) of eij. Next, si and sj connect to sk to form triangle and produce $\mathrm{CID}=1$ for this triangle.

On the arrival actuator IDs, the actuators in each cell choose active sensors in the cell to be Kautz nodes to make a complete $\mathrm{K}(\mathrm{d}, 3)$ graph.The KID assignment algorithm to select Kautz nodes to construct a Kautz graph. It has three steps:

The Kautz nodes connecting the actuators in a Kautz graph are chosen

The Kautz nodes connecting these selected Kautz nodes are chosen

The remaining Kautz nodes in the Kautz graph are chosen

\section{RESULTS AND DISCUSSION}

This section describes the node initiation, energy efficiency, data rate, lifetime. after a node initiation developed using algorithm, such initiation is called random node development.

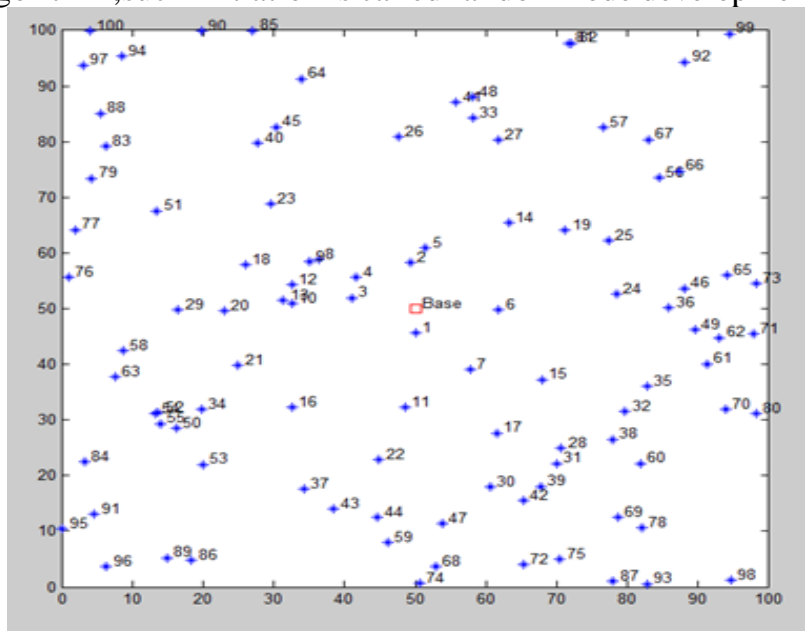

Fig.3.1.Node initiation (Random node Deployment)

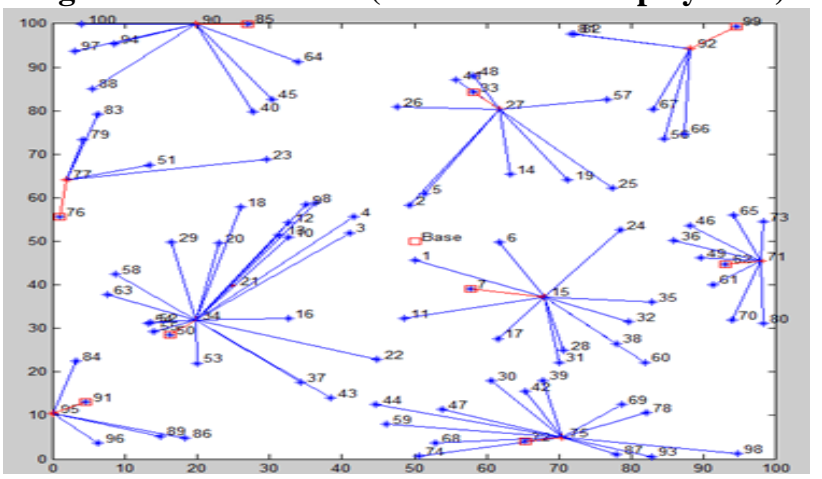

Fig.3.2. After Random nodeDeployment a.An Energy Vs Nodes 
The combination of nodes over the various degrees can be seen through the figure plotted above.

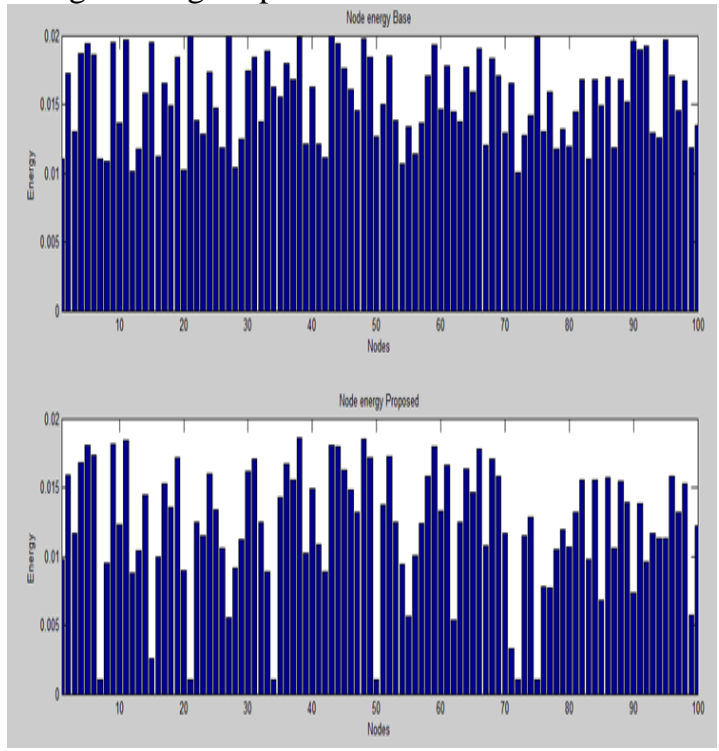

Fig..3.3. Energy vs Node

The above figure indicates the comparison of Base energy and the proposed energy. The proposed method produce large amount of energy compared to the base.

\section{b. Life Time}

A graph is plotted for the number of dead nodes against their rounds. Rounds symbolizes the life time of the nodes. Red lines are the ones from the base work. Green lines are what we have proposed in this project.

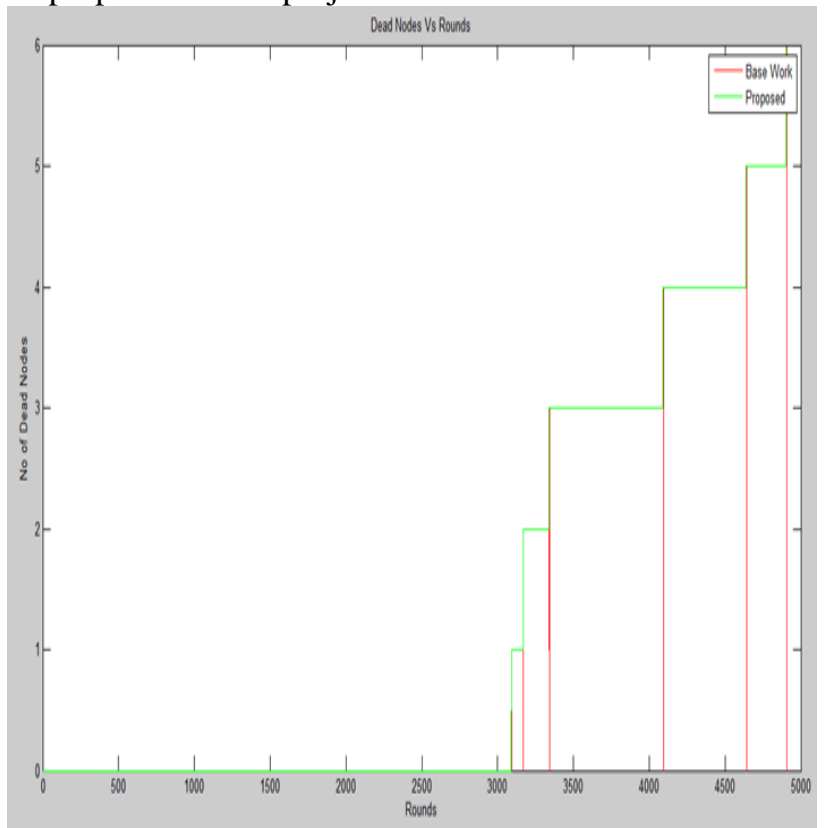

Fig.3.4. Life Time

\section{Data throughput}

Graph is plotted Data throughput against the rounds. First graph is for the base work and the second one is for the proposed. The proposed work provides high throughput of more than 4000 , where as the base work provides only 1000 plus results.

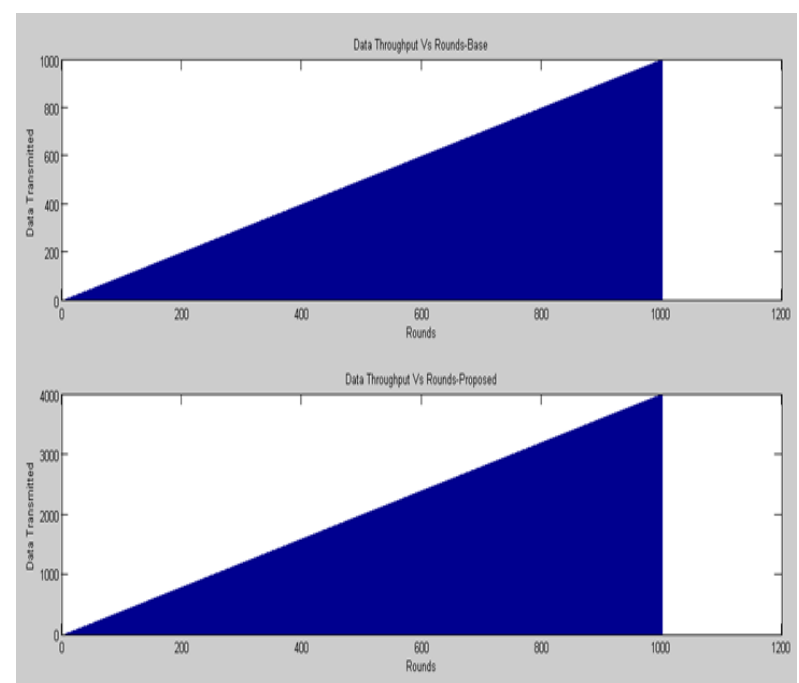

Fig.3.5. Data Throughput vs. Rounds

D.Average Energy

The graph shows the energy required for the formation of nodes. Base work required only $1.5 \mathrm{~kJ}$ where as in the proposed work it requires only $1.2 \mathrm{~kJ}$.

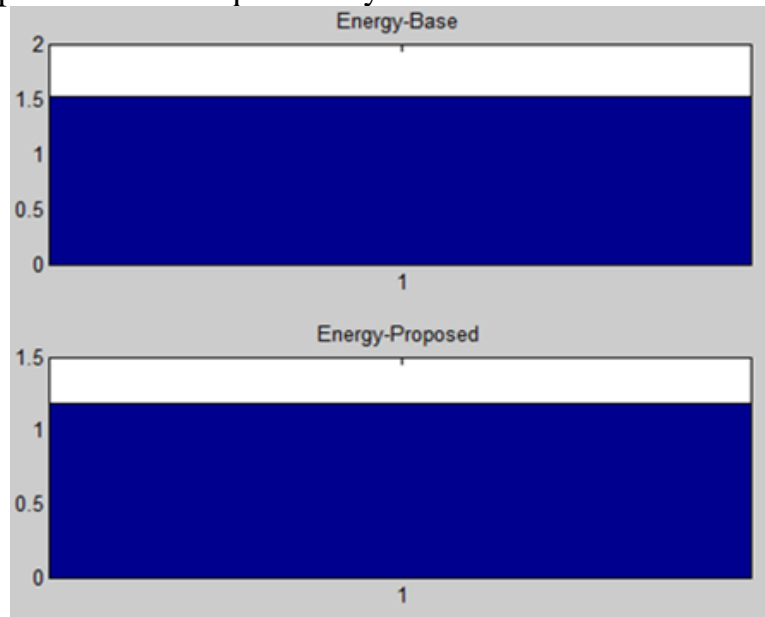

Fig.3.6. Average Energy

\section{CONCLUSION}

Energy-efficiency and fault-tolerance are seriousneeds for WSAN. Existing routing methods for WSANs does not fufill these needs. The properties of the Kautz graph show that it is an optimal topology for WSANs to meet the requirements. Proposed REFER method combines a Kautz graph protocol and an efficient fault-tolerant routing. This new topology is reliable with the physical topology, enabling real-time communication. Further, REFER influences DHT for the communication between Kautz-based cells for high scalability.

\section{REFERENCES}

1. L. F. Akyildiz and I. H. Kasimoglu. Wireless sensor and actor networks: research challenges. Ad hoc networks, 2(4):351-367, 2004.

2. J. A. Sanchez, P. M. Ruiz, and I. stojmenovic. Energy-efficientgeographic multicast routing for sensor and actuator networks. Computer Communications, Elsevier, 30(13):2519-2531, 2007.

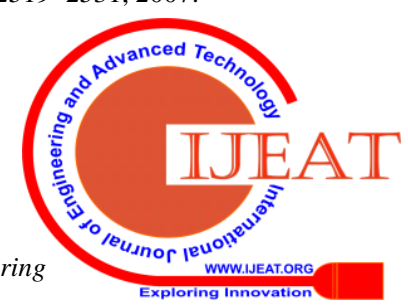


3. Dr.S.P.Anandaraj " Research Opportunities and Challenges of a Security Concerns associated with Big Data in Cloud Computing", IEEE International conference on ISMAC IoT in Social, Mobile, Analytics and Cloud published in IEEE DOI: 10.1109/I-SMAC.2017.8058278, Electronic ISBN: 978-1-5090-3243-3,Print ISBN: 978-1-5090-3242-6, INSPEC Accession Number: 17224835 on 10th to 11th Feb, 2017.

4. Ramesh, S 'An Efficient Secure Routing for Intermittently Connected Mobile Networks', 2017, Wireless Personal Communications, ISSN: 0929-6212 Vol.94, No.4, pp. 2705 - 2718.

5. E. Ngai, M. R. Lyu, and J. Liu. A real-time communication framework for wireless sensor-actuator networks. In Proc. of AC, pages 9-pp, 2006.

6. Kirubakaran, N. and Kathirvel, A. "A Unified Approach for detecting and eliminating selfish nodes in MANETS using TBUT", Springer-EURASIP Journalof on Wireless and Communications and Networking, Vol.143 DOI: 10.1186/s13638-015-0370x, 2015.

7. Dr.S.P.Anandaraj, S.Poornima,Sougandhika Reddy,M Sindhuja,P.Vedhakshatha, "Semantic Analysis On Communication And Security Issues Of Extensible Authentication Protocol (EAP) On Wireless Networks" International Journal of Advanced Computing and Electronics Technology(IJACET), ISSN(P):2394-3408,(O):2394-3416,Vol 2, No 1, January 2015

8. W. Hu, N. Bulusu, and S. Jha. A communication paradigm for hybrid sensor/actuator networks. In Proc. of PRIMC, volume 12, pages 47-59, 2004.

9. T. Melodia, D. Pompili, V. C. Gungor, and I. F. Akyidiz. A distributed coordination framework for wireless sensor and actuator networks. In Proc. of MobiHoc, pages 99-110, 2005.

10. C. Perkins, E. Belding-Royer, and S. Das. RFC 3561: Ad hoc on demand distance vector (AODV) routing, 2003.

11. Ramesh, S \& Indira, R, 'B-LoDiS Routing for Intermittently Connected MANET with Agent AES Approach', 2015, Internetworking Indonesia Journal, ISSN: 1942-9703 Vol.7, No.1 B, pp. 13-23.

12. Dr. S.P.Anandaraj, Dr.P.Manikandan, "Spot Detection For Morphological Convolution Using DNA Microarray", International Journal of Pure and Applied Mathematics, ISSN: 1311-8080 (printed version); ISSN: 1314-3395 (on-line version), SCIE 2013 Impact Factor= 7.19, Scopus $\mathrm{SJR}=0.322$, H-index=28,G-Index=48, Vol.118, Issue 14,pp.1-7,Feb 2018.

13. M. Caesar, M. Castro, E. B. Nightingale, G. O. Shea, and A. Rowstron. Virtual ring routing: network routing inspired by DHTs. In Proc. of Sigcomm, volume 36, pages 351-362, 2006.

14. A. Awad, R. German, and F. Dressler. P2P-based routing and data management using the virtual cord protocol. In Proc. of MobiCom, pages 443-444, 2006.

15. Ramesh, S \& Ganesh Kumar, P , 'Spray and wait routing with agents in intermittently connected MANETs', 2013, Journal of Artificial Intelligence, ISSN 1994-5450, vol. 6, no. 2, pp. 123-133.

16. H. Shen, Z. Li, and K. Chen. A Scalable and Mobility-Resilient Data Search System for Large-Scale Mobile Wireless Networks. TPDS, 2013.

17. Kirubakaran, N. and Kathirvel, A. "Performance Improvement of Security Attack in Wireless Mobile Ad Hoc Networks", Research Journal of Asian Journal of Information Technology, Vol.13, No.2, pp. 68-76, 2014.

18. D. Guo, J. Wu, H. Chen, and X. Luo. Moore: An extendable peerto- peer network based on incomplete kautz digraph with constant degree. In Proc. of Infocom, pages 821-829, 2007.

19. D. Guo, Y. Liu, and X. Ki. Bake: A balanced kautz tree structure for peer-to-peer networks. In Proc. of Infocom, 2008.

20. Kirubakaran, N. and Kathirvel, A. "Cross Layer Umpiring System Design in Wireless Mobile Ad Hoc Networks", Asian journal of Science and Technology, Vol. 08, No.04, pp. 4644-4646, 2017.

21. K. Zuo, D. Hu, H. Wang, Q. Wu, and L. Su. An efficient clustering scheme in mobile peer-to-peer networks. In Proc.of ICOIN, pages 1-5, 2008.

22. Z. Li and H. Shen. A Kautz-based Real-time and Energy-Efficient Wireless Sensor and Actuator Network. In Proc. of ICDCS, pages 1-10, 2012.

23. D. B. Johnson and D. A. Maltz. Dynamic source routing in ad hoc wireless networks. Mobile Computing, 353:153-181.

24. Dr.S.P.Anandaraj, D.Naveen Kumar, S.Poornima, "Invariant Character View Recognition in Artificial Neural Networks based on pattern classification" Global Journal of Computer Science and Information Technology (GJCSIT), Vol 1, No.1,pp. 33-37, Oct 2014.

25. Kirubakaran, N. and Kathirvel, A. "Secured Unicast Routing using Cross layer design in Wireless Mobile Ad Hoc Networks", The international journal of Forensic Computer Science, DOI: 10.5769/J201701002, 2017

26. Ramesh, S \& Ganesh Kumar, P, 'A secure 3-way routing protocols for intermittently connected mobile ad hoc networks', The Scientific World Journal, ISSN: 1537-744X, July 2014, vol. 2014, Article ID. 865071, pp. 1-13, DOI: $10.1155 / 2014 / 865071$

27. N. Nithiyanandam, K. Venkatesh, M. Rajesh, Transfer The Levels Of The Monitored Carbon, Nitrogen Gases From The Industries, International Journal of Recent Technology and Engineering, Volume-7 Issue-6S3 April, 2019.
28. Sivanesh Kumar, A., Brittoraj, S., Rajesh, M., Implementation of RFID with internet of things, Journal of Recent Technology and Engineering, Volume-7 Issue-6S3 April, 2019.

29. Rajesh, M., Sairam, R., Big data and health care system using mlearningJournal of Recent Technology and Engineering, Volume-7 Issue-6S3 April, 2019.

30. Rajesh, M., and J. M. Gnanasekar. "Path Observation Based Physical Routing Protocol for Wireless Ad Hoc Networks." Wireless Personal Communications 97.1 (2017): 1267-1289. 